La

Révolution

française

\section{La Révolution française}

Cahiers de l'Institut d'histoire de la Révolution française

5 | 2013

Le républicanisme anglais dans la France des

Lumières et de la Révolution

\title{
Histoire républicaine et conscience révolutionnaire
}

Les enjeux politiques de la traduction de Catherine Macaulay

\section{Francesco Dendena}

\section{OpenEdition}

\section{Journals}

Édition électronique

URL : http://journals.openedition.org//rf/949

DOI : $10.4000 / / r f .949$

ISSN : 2105-2557

Éditeur

IHMC - Institut d'histoire moderne et contemporaine (UMR 8066)

\section{Référence électronique}

Francesco Dendena, «Histoire républicaine et conscience révolutionnaire », La Révolution française [En ligne], 5 | 2013, mis en ligne le 31 décembre 2013, consulté le 19 avril 2019. URL : http:// journals.openedition.org//rf/949 ; DOI : 10.4000/Irf.949

Ce document a été généré automatiquement le 19 avril 2019

(C) La Révolution française 


\title{
Histoire républicaine et conscience révolutionnaire
}

\author{
Les enjeux politiques de la traduction de Catherine Macaulay
}

Francesco Dendena

1 Varennes marque un moment de crise majeure dans la Révolution française. Entre la fin du mois de juin 1791 et le massacre du Champs-de-Mars, la France vit dans un interrègne, ni monarchie ni république où l'unité du côté gauche vole en éclat. La fuite de Louis XVI oblige les révolutionnaires à repenser le système qu'ils avaient mis en place les deux années précédentes et surtout à s'interroger sur la place du roi dans le nouveau régime : peut-on régénérer un monarque félon ou faut-il le punir? Tandis que la majorité veut croire que la première solution est réalisable, très vite émerge une poignée de républicains qui critique la politique du compromis. L'arme de leur critique ? L'histoire. Leur hantise? La guerre civile anglaise du XVII siècle. Pour ces hommes, face à un roi traitre qui s'est élancé à la vaine poursuite de l'Ancien Régime, il ne fait pas de doutes que la nation doit avoir le courage de lire l'histoire moderne et en tirer les conclusions qui s'imposent: derrière Louis se cache un Charles $\mathrm{I}^{\mathrm{er}}$, tout comme derrière celui-ci se dissimulait un Charles IX. L'histoire des rois ne raconte qu'un récit, celui du despotisme ${ }^{1}$.

2 Avant d'arriver à l'Assemblée nationale, c'est à la société des Amis de la Constitution que les débats sur le sujet sont les plus vifs. Le souvenir de l'expérience du Commonwealth, d'abord sous-entendu, puis utilisé comme repoussoir de ce qui ne peut être nommé, au fil des jours s'impose dans les débats. Jacques-Pierre Brissot est le premier y à faire référence de façon positive en faisant de l'Angleterre délivrée de son roi, un modèle de patriotisme bien digne d'être imitée. Pourtant c'est son ami et collaborateur au Patriote Français, Joseph-Marie Girey-Dupré, qui a le courage de déchirer complètement le voile lorsqu'il lance, devant un public médusé :

Deux fois les Anglais vous ont donné un grand exemple: vous êtes dignes de l'imiter. On vous a dit que ce peuple témoignait tous les ans sa douleur d'avoir été trop rigoureux : que l'on sache que les aristocrates seuls, et les esclaves, célèbrent la mémoire du prétendu martyr Charles I, et que tous les patriotes ne le regardent que comme le martyr de sa fierté tyrannique et de son despotique entêtement ${ }^{3}$. 
Dans ce discours, le souvenir du XVII ${ }^{e}$ siècle anglais est cité, au sens plein du terme, en tant qu'exemplum pour suggérer ce qui reste encore à faire. À ce titre l'on pourrait reprendre les considérations élaborées par Pierre Serna ou celles d'olivier Lutaud, qui, à propos d'autres emprunts et d'autres échanges autour de l'expérience du Commonwealth, ont parlé d'un souvenir tourné dynamiquement vers l'avenir: sans doute les mots de Girey-Dupré ne font que confirmer la justesse de ces deux lignes interprétatives ${ }^{4}$.

4 Toutefois, ces interventions suggèrent aussi de réinterroger de façon plus générale la question de la permanence du souvenir du XVII ${ }^{e}$ siècle anglais et de son exploitation politique lors de la Révolution de 1789: permanence longtemps oubliée, ou plutôt présence longtemps niée ${ }^{5}$, car à contrecourant de deux mythes nationaux, construits sur le registre de l'altérité dont la meilleure preuve aurait justement été la différence radicale de deux expériences révolutionnaires ${ }^{6}$.

5 Les historiographies du XIX et du XXe siècle n'ont fait que reprendre le sillage tracé, pour des raisons éminemment politiques, par les contemporains de la Révolution française, fussent-ils à Londres ou à Paris, fussent-ils ses opposants ou bien ses partisans. Au fond, l'importance des Réflexions sur la Révolution de France d'Edmund Burke en 1790 résidait moins dans leur originalité que dans la capacité de renverser et transformer en une puissante critique ce que de leur côté les révolutionnaires avaient revendiqué dès l'écroulement de l'absolutisme comme leur plus grande mérite ${ }^{7}$ les principes métahistoriques et universels. C'est donc une perspective unanime donc que celle d'une Révolution interprétée comme négation du temps, perspective qui implique forcement le corollaire d'une Révolution sans précédents. Admettre le contraire revenait à dire qu'elle n'était qu'une étape, qu'elle appartenait à un devenir historique dont l'idée contenait déjà en elle-même un principe de perfectibilité qui aurait nié sa légitimité8. Ainsi, le concept de tradition révolutionnaire serait un oxymore d'une portée épistémologique limitée, dans la mesure où il dévoierait la volonté qui était celle des révolutionnaires, en empêchant la compréhension de leur horizon d'action.

6 Pourtant, le refus de l'histoire en tant que code normatif et inspirateur de l'action révolutionnaire, bien réel et précocement formulé, n'était-t-il pas une invitation à récrire le passé plutôt que l'effacer? N'était-t-il pas, au fond, un constat ? Le constat du fait que, face à l'écroulement du despotisme, l'histoire dans la forme où elle avait été écrite jusqu'à ce moment, était incapable de rendre compte de l'accélération du temps dont la Révolution était la preuve car elle était le récit d'un régime qui n'existait plus. Non seulement cette affirmation contient l'aveu de sa non réalisation, mais aussi et surtout, elle confirme l'urgence de changer de paradigme historiographique lorsqu'un nouveau régime d'historicité surgit ${ }^{9}$.

7 Cela est d'autant plus évident à partir de Varennes, lorsque l'identité entre progrès historique et cours de la Révolution, qui avait justifié l'impossibilité de toute comparaison avec les autres crises du passé, perd sa validité interprétative. Dès ce moment, la régénération placée sous le signe de l'incomparable, se poursuit au miroir d'une histoire nouvelle qui reste encore à écrire. Et ce n'est pas un hasard si, du républicain Lavicomterie, « assis au milieu des débris de ces lois vandales, de ces usages ridicules et barbares sous lesquelles la France a gémi quatorze siècles", au Feuillant Barnave, qui prend acte de la "pulvérisation » du monde ancien, pour parvenir à Rabaud de SaintEtienne, les révolutionnaires eux-mêmes s'efforcent de «mémoriser la Révolution " pour reconstruire une narration, un ordre temporel susceptible de donner un sens à la rupture à 
laquelle ils ont participé ${ }^{10}$. Pour paraphraser la célèbre phrase de Friedrich Schlegel, ils deviennent des "prophètes tournés vers le passé » pour établir leur légitimité. Le refus de faire de l'histoire un code normatif et inspirateur dévoile alors pleinement sa signification: il est une invitation non pas à effacer le passé mais à le révolutionner, à " réorganiser l'histoire de France à l'avantage de la nation ${ }^{11}$. »

Revenons sur les mots de Girey-Dupré : «Les aristocrates seuls, et les esclaves, célèbrent la mémoire du prétendu martyr Charles I [...] tous les patriotes ne le regardent que comme le martyr de sa fierté tyrannique. » Pour le futur conventionnel, comme pour Brissot, pardelà des siècles, par-delà des frontières, le souvenir des luttes du XVII ${ }^{e}$ siècle anglais acquiert une valeur métahistorique qui structure le débat contemporain: dans leurs discours, l'histoire se transforme en une expérience du passé qui définit une conscience politique présente. Ces interventions sont-elles des cas isolés? Ou bien, peuvent-elles révéler l'occasion pour étudier la présence du souvenir de «l'arbre de la liberté » planté « au milieu des torrents de sang » en Angleterre ${ }^{12}$ ?

Comment les expériences républicaines et révolutionnaires du XVII ${ }^{e}$ siècle anglais, plutôt que les idéaux, dont l'importance comme source d'inspiration a été récemment prise en compte ${ }^{13}$, sont réintégrées dans le contexte révolutionnaire français? Ces pages seront l'occasion de penser la construction et l'articulation d'un discours politique à partir de la réflexion sur le passé et comprendre, au prisme d'une histoire intellectuelle transnationale, dans quelle mesure le souvenir des crises politiques du passé est récupéré, voire clairement dévoyé sous l'empreinte de la dialectique formée par les circonstances et par la volonté politique des acteurs culturaux. Si les débats qui ont eu lieu à l'occasion de la fuite de Varennes ont été l'occasion de problématiser la thématique, celle-ci sera dorénavant développée à travers l'analyse de la réception en France de l'ouvrage de Catherine Macaulay, The History of England from the accession of James I to the Elevation of the House of Hanover, qui est proposé (de manière incomplète) au public français entre début 1791 et fin 1792 grâce à l'intervention de Honoré Riqueti de Mirabeau et de ses collaborateurs $^{14}$.

Le choix de ce texte s'explique d'abord par l'influence qu'il a en tant qu'outil culturel et politique pour conceptualiser la rupture révolutionnaire dans un contexte d'Ancien Régime et ensuite pour l'importante valeur politique qu'il acquiert lors de sa traduction. Comme l'écrit en février 1792 un rédacteur anonyme du Moniteur national en recensant la publication des premiers tomes de l'Histoire d'Angleterre, «tous les amateurs de l'histoire, et surtout les âmes libres, doivent s'empresser de se procurer ces deux volumes, et attendre avec impatience la publication des six [autres]» car l'œuvre est "l'une des plus importantes jamais publiées depuis la Révolution [de 1789 $]^{15}$.» Certes, l'Histoire d'Angleterre est loin d'être le seul écrit à faire référence aux événements du XVII ${ }^{\mathrm{e}}$ siècle anglais publié lors de la Révolution de $1789^{16}$, toutefois il est sans doute le plus significatif en étant le résultat d'un travail éditorial complexe et politique qui s'étale sur plusieurs années. Le texte s'enrichit progressivement de significations différentes, parfois contradictoires, qui varient non seulement en fonction des sensibilités politiques des curateurs qui se succèdent au fil du temps, mais, installé au milieu d'un changement de régimes d'historicité et tiraillé par une accélération hors pair, le récit républicain élaboré par Macaulay se trouve être le prisme à travers lequel lire l'histoire d'une nouvelle révolution.

11 L'existence de cette traduction n'est pas inconnue; le texte a été examiné récemment pour être replacé dans le contexte plus général de la production politique du député de la 
Constituante ${ }^{17}$. De nombreux aspects concrets, relatifs au travail éditorial restaient à éclairer; tandis que les interventions précédentes avaient conçu la traduction comme l'aboutissement d'un riche dialogue entre le tribun et le passé anglais, ces pages en revanche s'efforcent de saisir l'ensemble des interventions dont l'histoire de Macaulay a été l'objet pour avancer une nouvelle hypothèse interprétative concernant les relations qui se nouent entre histoire, identité politique et conscience révolutionnaire.

Cette perspective d'étude explique par ailleurs la structure de l'article qui, en s'appuyant sur les études anglo-saxonnes, consacre son ouverture au travail de l'historienne et à sa réception dans le contexte politique anglais. Ce préalable se révèlera ensuite essentiel pour comprendre les raisons de son passage en France et pour expliquer surtout l'importance qu'il aura plus tard.

\section{1. «To do justice our ancestor »}

13 Salué en France en tant que modèle politique alternatif à l'absolutisme ${ }^{18}$, en Angleterre, le compromis instauré par la Glorieuse Révolution est l'objet d'âpres débats tout au long du siècle. Autant cette rupture est considérée par certains, comme un pacte garant de la stabilité politique du pays, autant elle est remise en cause par d'autres qui la considèrent en revanche comme une occasion ratée ou, pire, une trahison des libertés anglaises fondamentales. Dans ce contexte politique tendu, l'écriture de l'histoire nationale constitue un véritable enjeu, car s'interroger sur la nature et la signification de ce qui s'est passé en 1688 signifie porter un jugement à la fois sur ce dont elle est l'aboutissement, et sur le système qui en a résultée ${ }^{19}$. En ce sens, l'historien, qu'il le veuille ou non, est un acteur politique à part entière de la communauté à laquelle il appartient.

Le sort de l'History of England de David Hume, publiée entre 1754 et $1762^{20}$, illustre bien ce propos. Dès sa sortie et en dépit des intentions de son auteur, l'ouvrage suscite une vaste polémique, rapidement perçue comme une remise en cause du socle mémorial commun du royaume, construit à son tour autour de la vulgata whig de l'histoire, légitimant l'équilibre social et politique en place. En effet, le récit élaboré par le philosophe constitue un démenti de l'existence historique d'une ancienne constitution, dépôt des traditionnelles libertés anglaises et par là, nie le caractère défensif et réactif de l'action du Parlement lors de la guerre civile. Selon le philosophe, celle-ci ne serait pas provoquée par le despotisme royal, mais elle serait plutôt la conséquence d'un changement des rapports de forces dont le principal bénéficiaire aurait été la nation, qui par la suite se serait imposée sur la monarchie par l'action de ses représentants. La seule faute de celleci aurait été de ne savoir ni s'adapter ni gouverner ce processus, dont au final elle avait été la victime. En postulant l'idée d'une guerre civile fille du progrès et des circonstances, Hume soustrait aux vainqueurs non seulement la légitimité qui leur vient du passé, en tant que défenseurs des libertés traditionnels ${ }^{21}$, mais ce qui est plus important, il renverse même le présupposé fondamental de la Glorieuse Révolution qui faisait reposer la légitimité de tout gouvernement sur son origine contractuelle et le respect des droits de la nation. Certes, le compromis de 1688 est « one of the Finest and the Most Subtile invention ", mais il l'est simplement parce qu'il a assuré un terme à l'instabilité qui avait caractérisé les décennies précédentes ${ }^{22}$. Pour Hume, le seul titre de mérite d'un régime réside exclusivement dans sa capacité à assurer dans la durée la stabilité et la paix interne ${ }^{23}$. Or, c'est justement pour démentir cette vision, très vite et à tort qualifiée de tory history ${ }^{24}$, que, à peine un an après le dernier tome du travail de Hume, parait The History of 
England from the accession of James I to the Elevation of the House of Hanover de Catharine Macaulay. Ce que le philosophe écossais considérait comme une période de trouble et d'anarchie, dominée par le fanatisme religieux, sous la plume de l'historienne devient «the finest hour» de l'histoire anglaise où la nation, menée par ses exposants les plus valeureux, a fait plier et a puni un souverain despotique au nom de ses anciens droits. En effet, l'historienne réinscrit le conflit du XVII ${ }^{e}$ siècle à l'intérieur d'une tension pluriséculaire qui oppose la nation anglaise au pouvoir monarchique, lequel avait perverti ses droits fondamentaux et imprescriptibles depuis la conquête normande. Macaulay relit ainsi la guerre civile dans des termes antinomiques qui se chargent de connotations morales dans la mesure où la réaction patriote, au nom de la défense des libertés traditionnelles, serait le fruit d'un processus de régénération morale de la nation mené sous la houlette du Long Parlement, dépositaire de sa conscience collective. Cette lutte révèle l'importance de l'enjeu : l'esclavage ou la liberté. La guerre civile, dans cette perspective, ne serait plus le produit d'une histoire nationale, comme le prétendait Hume, mais bien au contraire le résultat de la volonté d'une minorité qui, en se sacrifiant pour le bien public a été capable de donner un exemple de vertu en modifiant le cours de l'histoire. Dans cette logique, Macaulay justifie non seulement l'opportunité du régicide, mais elle fait du Commonwealth et du moment républicain le seul véritable moment de liberté de l'histoire anglaise. Si l'expérience républicaine a échoué, ce serait moins à cause des agissements de Cromwell, que de la faiblesse de la vertu du peuple anglais ${ }^{25}$.

Étalé sur un espace de plus de vingt ans et élaboré à partir d'une profonde connaissance des sources originales ${ }^{26}$, l'ouvrage de Macaulay s'éloigne de l'interprétation de Hume, moins dans ses conclusions historiographiques que dans la redéfinition des rapports entre espace public et histoire, suggérant une autre conception de l'action politique ${ }^{27}$. Tandis que pour Hume, comme en général pour l'historiographie des Lumières, l'histoire est le produit d'une transformation dont il faut rendre compte, pour Macaulay, plus proche sur ce point d'une historiographie humaniste et classique, elle demeure le résultat d'une tension morale permanente qui s'incarne, au gré des circonstances, dans des acteurs qui sont porteurs de valeurs métahistoriques transcendantes. En dernière analyse, le véritable moteur du changement historique n'est pas le progrès en soi, mais la volonté humaine pour soi, individuelle ou bien collective ${ }^{28}$.

De cette vision découle à la fois une méthode, qui fait de l'explication et de l'interprétation des caractères moraux des protagonistes l'un des fondements épistémologiques du récit historique, et une conception de l'écriture historique pensée comme acte de jugement et non pas d'entendement ${ }^{29}$. Certes, les faits doivent être racontés de manière impartiale sur des bases scientifiques, mais il ne demeure pas moins que l'historien, à la lumière d'une instance métahistorique supérieure, ne peut se soustraire à une tâche interprétative censée révéler à son public dans quelle mesure les protagonistes des faits ont contribué à la cause de la raison et la libertée ${ }^{30}$. Ce qui revient à postuler la nécessité d'une prise de position partisane de la part de l'auteur. Dès lors, l'histoire devient un " primary medium in the political debate » : elle fonde et crée une conscience collective de la liberté en constituant un socle sur lequel bâtir la réforme d'un système politique, jugé profondément corrompu. Loin de fonder une idéologie au sens propre de la parole, elle permet néanmoins de penser un modèle d'engagement alternatif au service de la communauté, construit autour de l'idée de vertu ${ }^{31}$. Au final pour Catherine Macaulay, l'écriture de l'histoire constitue le préalable et la forme plus achevée de tout engagement politique car, par le biais des exemples du passé, elle réveille la 
conscience de la nation et l'éduque à la liberté non pas pour imiter ce qui a été fait selon une conception cyclique du temps, mais pour rendre possible le changement, au nom des valeurs dont elle se fait porteuse.

Individuals may err, but the public judgment is infallible. They only want a just information of facts to make a proper comment. Labor to attain truth, integrity to set it in its full light, are indispensable duties in an historian ${ }^{32}$.

Loin d'être spécifique à Macaulay, cet usage politique de l'histoire est l'héritage d'une longue tradition qui remonte à la fin de XVII siècle, lorsque le mouvement real-whig avait trouvé dans le passé le langage et les exemples en mesure de remettre en cause la légitimité du compromis établi par la Glorieuse révolution, conçu comme une trahison des valeurs morales et politiques défendus pendant la guerre civile par les patriotes ${ }^{33}$. Tout au long du XVIII ${ }^{e}$ siècle, l'histoire récente avait été constamment utilisée pour dénoncer le patronage du ministère et de la Cour sur le parlement, le maintien d'une armée professionnelle sur le sol national et enfin la création d'une dette nationale lourde de conséquences sur les choix politiques contemporaines. C'est une voix éminemment critique que celle du passé, seul antidote pour réveiller la nation de sa léthargie, de la stabilité dont Hume avait tissé les éloges et qui ne serait que l'aboutissement final d'un processus d'asservissement collectif.

The form of the constitution may be preserved, when the spirit of it is lost; and nothing is more likely to happen, whilst those persons who are appointed to maintain it are ignorant of those fundamental principles, where on the barriers which defend civil liberty from prerogative are founded. [...] Corruption, that undermining mischief, had sapped the foundation of a fabric, whose building was cemented with the blood of our best citizens ${ }^{34}$.

En écrivant ces mots, Macaulay fait de sa plume un écho, en fusionnant son activité de savante dans une collectivité politique real-whig, dont elle partage les positions et dont elle est une figure très active dans les années $1760-1770^{35}$. Il est aisé au final de comprendre pourquoi, dans un contexte politique marqué par les débats intenses suscités par le mouvement wilkite et par une mobilisation extraparlementaire favorable à une réforme constitutionnelle en un sens démocratique ${ }^{36}$, l'œuvre de Macaulay acquiert vite une vaste renommée, comme en témoignent à la fois son important succès commercial et la grandissante visibilité de son auteure ${ }^{37}$. En effet, The History of England transforme Catharine Macaulay en une figure célèbre, sinon importante, de la scène politique anglaise en marquant le début d'un engagement publique qui ne s'achèvera qu'avec sa mort en mars 1791. De l'indépendance de la Corse à la cause américaine, du soutien à Wilkies jusqu'à la polémique avec Burke en défense de la Révolution française, en passant des combats pour l'éducation des femmes, Catherine Macaulay devient une inlassable critique des limites de la monarchie anglaise au nom d'une liberté civique et politique qu'elle considérait indissociable d'un discours moral et républicain et dont les meilleurs exemples avaient été « the exalted patriots who have illustriously figured in this country 38.»

\section{Brissot et Macaulay}

Rien d'étonnant alors si, quelques années plus tard ses ouvrages attirent l'attention des expatriés français qui se trouvent en Angleterre, souvent en contact avec les cercles radicaux, d'autant que le conflit qui oppose la métropole aux colonies américaines est censé être la meilleure preuve du processus de corruption politique du système anglais 
dont les origines avaient été découvertes par Macaulay dans les événements du siècle précédent.

Dès 1780, Jacques-Pierre Brissot avait repris les critiques real-whigs dans son Testament politique de l'Angleterre en dénonçant la corruption et la dette publique comme des véritables sources de l'impérialisme anglais ${ }^{39}$, mais c'est seulement plus tard, à l'occasion de son deuxième séjour en Angleterre, qu'il étudie le système politique de l'île, connaissant par là même occasion Macaulay et ses ouvrages ${ }^{40}$. Ceux-ci deviennent les points d'appui pour étayer une vision de la constitution britannique critique et alternative à celle répandue sur le continent par « le livre de Delolme, qui [en effet n'est] qu'un ingénieux panégyrique ${ }^{41}$. » En particulier, en 1786 lors de sa traduction des Lettres philosophiques et politiques sur l'histoire de l'Angleterre d'olivier Goldsmith, Brissot choisit d'ouvrir son travail en reprenant des longs extraits de l'introduction de The History of England et il annonce même avoir « corrigé » le travail qu'il vient de publier en s'appuyant sur l'autorité de "l'historienne républicaine ${ }^{42}$."

21 À quoi donc l'Histoire sera-t-elle utile? Aux Peuples : elle doit leur révéler leurs droits, la manière dont ils les ont perdus, le chemin qui les a conduits de la liberté à l'esclavage, le chemin contraire qui peut les ramener de l'esclavage à la liberté, en un mot elle doit leur apprendre sous quel Gouvernement, sous quelles Lois l'homme a été plus grand plus heureux sous quel Gouvernement, il est ce qu'il doit être. [...] L'Histoire ne peut être utile au peuple que sous le rapport politique; il en résulte que celle qui lui présente le tableau le mieux entendu de ces révolutions heureuses qui ont perfectionné des Gouvernements doit être plus instructive, plus intéressante, et doit être préférée ${ }^{43}$.

Selon Brissot, l'histoire d'Angleterre doit être "préférée à presque toutes les Histoires modernes ", car elle permet de saisir « ce qui distingue un peuple libre, d'une populace ou d'une meute d'esclaves, ce qui peut rendre une nation heureuse.» Autrement-dit, elle permet de penser la problématique de la liberté politique, en termes de conquête et de lutte ${ }^{44}$. D'ailleurs, le long parallèle que Brissot trace entre Hume et Macaulay, les « deux [seuls historiens] qui méritent d'être nommés ", se résout à l'avantage de la dernière en raison de la valeur performative que son récit peut acquérir dans la Révolution ${ }^{45}$.

En effet, au-delà des nombreuses citations qu'il tire de l'ouvrage de Macaulay, Brissot se montre profondément influencé sur le fond par sa conception de l'histoire en tant que moyen éducatif, tout en adaptant cette approche au contexte français. Il ne se limite pas à reprendre presque en entier l'introduction de l'historienne, mais il en modifie la perspective, en lui attribuant un sens nouveau par l'intégration d'une dimension humaniste, enchâssée dans une vision du devenir historique proche de celle des Lumières, où la notion de progrès joue un rôle décisif ${ }^{46}$. Les choix lexicaux opérés par le futur conventionnel sont en ce sens tout-à-fait révélateurs de ses convictions sur le plan politique et philosophique. Comme il l'expliquera plus tard, «il fallait [...] ne regarder l'Histoire de Macaulay que comme un dépôt de matériaux précieux » pour en tirer " un ouvrage nouveau adapté à notre goût et à nos besoins ${ }^{47}$. »

Alors que Macaulay considère la liberté politique en tant que redécouverte d'un pacte originaire et conçoit son histoire comme un récit moral qui, par la présentation des exemples des patriotes du passé rend possible une amélioration de la société, le futur révolutionnaire trouve dans le passé la possibilité de saisir les conditions et les modalités du changement. L'opportunité se présente de saisir la Révolution comme objet historique en développant un discours générateur de la liberté. Tandis que l'ouvrage de Macaulay 
avait été écrit afin de permettre une régénération patriotique au nom de la tradition, il est lu par Brissot comme une histoire de la nouveauté et de la rupture qui s'inscrit dans une logique de progrès. La liberté, conçue comme affranchissement, devient un processus en devenir, résultat des connaissances et surtout des « orages » qui ont émaillé le passé de l'île en permettant l'élaboration d'une Constitution, qui dans ses principes fondateurs représentait un modèle pour les autres Etats d'Europe.

« Des écrivains peu éclairés et d'une politique étroite, ont plaint l'Angleterre d'avoir éprouvé tant d'orages. Sans doute les Anglais eussent été heureux plutôt si on leur accordé le bien qu'ils ont été forcés de conquérir; mais malgré ce qui leur est arrivé, les croit-on plus à plaindre que ces nations [...], dont l'existence n'est qu'un continuel esclavage, et qu'un long calme conduit de l'assoupissement à la mort [ ?] $]^{48}$

Brissot n'est pas le seul français à s'intéresser à l'histoire de Macaulay au milieu des années 1780-1790. L'œuvre attire aussi l'attention de Mirabeau, qui, à la même époque, a fait de Londres son refuge. L'auteur de l'Essai sur le despotisme ne se limite pas à étudier attentivement le système constitutionnel du Pays, mais entre 1784 et 1785 il fréquente la scène politique anglaise, en nouant notamment d'étroites relations avec les milieux radicaux, dont il reprend l'approche critique ${ }^{49}$. Il arrive même à la conclusion que, «l'harmonie qui a longtemps existé parmi les ordres de l'Etat » est désormais dissoute à l'avantage du pouvoir central ${ }^{50}$. Pour le futur Constituant, c'est de l'autre côté de l'Atlantique, parmi «les Américains, ces généreux disciples des indépendants que détruisit le malheureux et infâme Cromwell » qu'il faut chercher les héritiers des parlementaires qui se battirent pour refonder le pacte social au prisme de la libertées.

C'est au flambeau de la raison de ces hommes éminemment éclairés dans un siècle où personne ne l'était, que les Américains ont allumé la leur. Pour s'en convaincre, on n'a qu'à lire l'excellente histoire de mademoiselle Macaulay [sic], et comparer ce que cette excellente femme rapporte sur les settlers [sic], dont elle a si bien développé les principes, à tous les écrits publics des Américains durant les troubles 52 .

Ce passage, tiré d'une lettre de juillet 1786 adressée à Brissot est doublement révélateur, en illustrant du même temps l'attention que Mirabeau porte à l'ouvrage de Macaulay et la conception de l'histoire du futur tribun, proche de celle de la «Portia de l'Angleterre » pour laquelle il montre une profonde admiration ${ }^{53}$. Ce texte permet de mieux comprendre la genèse de la traduction et la valeur politique qui lui est attribuée.

En effet, quoique la version française de l'ouvrage ait été éditée six mois après sa mort survenue en avril $1791^{54}$, Mirabeau conçoit l'idée de traduire The History of England de Macaulay avant $1785^{55}$. C'est à cette occasion que, pleinement conscient de la valeur commerciale de l'œuvre, il s'active pour trouver quelqu'un qui puisse se charger du travail à sa place tout en gardant ferme l'intention de la faire paraître à son propre nom ${ }^{56}$. En dépit de ce qu'affirme Brissot dans ses Mémoires ${ }^{57}$, ce n'est pas à lui que Mirabeau s'adresse pour réaliser la tâche mais, dès le départ, il pense plutôt à Jean-Baptiste Durival et à Charles-Philippe Guiraudet, deux hommes de lettres qu'il connaît déjà bien et qui plus tard, durant la Révolution, seront associés à son célèbre atelier de travail ${ }^{58}$.

L'ouvrage a été effectivement traduit dans son intégralité par le second quelques années plus tard tandis que Mirabeau se limite à écrire, probablement dans la première moitié de 1788, un long discours préliminaire qui retrace toute l'histoire de l'île depuis l'antiquité jusqu'à l'événement de la maison des Stuart ${ }^{59}$. En ce sens l'Avis de l'éditeur, placé au début de l'ouvrage et qui lui attribue aussi la traduction des deux premiers tomes est un mensonge explicable pour des raisons purement commerciales. En revanche, ce 
témoignage aide à fixer une date limite assez précise pour les interventions de Mirabeau sur le texte, achevées avant son départ dans le Midi lors des élections pour les États Généraux ${ }^{60}$.

Loin de constituer un détail, la datation de la préface se révèle un point décisif, si l'on sait qu'au cours de l'année 1789 Mirabeau fait paraître sous son propre nom un traité Sur la liberté de la presse, imité de l'anglais de Milton et qu'ensuite il fait traduire par l'un de ses collaborateurs, un autre ouvrage du grand poète républicain anglais: la Pro Populo anglicano defensio ${ }^{61}$. Cette vision d'ensemble permet de saisir, au-delà de l'intérêt, jamais absent des pratiques du noble désargenté, la traduction de l'histoire de Macaulay, dans un projet cohérent. Ce dernier vise à récupérer des textes de la tradition républicaine anglaise censés éclairer la crise du royaume. Comme il l'aurait dit lui-même à la fin de 1788, lorsqu'il fut obligé d'abandonner définitivement l'œuvre de Macaulay pour la confier aux soins de Guiraudet :

Cette traduction n'est pas dans nos circonstances, un ouvrage ordinaire. Il existe tant de points de contact et de rapport entre ces événements, ces personnages et nous, qu'en se bornant à les indiquer dans de simples notes, on se trouvera faire l'histoire des deux révolutions ${ }^{62}$.

L'intérêt de Mirabeau pour l'histoire ne saurait surprendre, comme des analyses récentes l'ont démontré, c'est justement à travers l'étude du passé que le futur Constituant a construit sa grammaire politique et sa posture critique à l'égard de la monarchie française. Pour reprendre l'expression de François Quastana, "l'histoire nationale [a constitué] pour Mirabeau le creuset de sa réflexion politique », dès les années $1770^{63}$.

Elle lui a permis de concevoir l'absolutisme sous l'aspect d'une construction historique, fruit d'une tendance usurpatrice de la part du pouvoir monarchique ayant perverti l'équilibre des ordres de l'époque carolingienne, en confisquant les droits de la nation. Selon cette perspective, l'histoire de France devenait le récit d'une lente décadence qui s'accompagne d'une succession de bouleversements, imposés par le despotisme à une communauté soumise au bon vouloir de ses gouvernants car ignorante de ses droits. En effet, plus qu'affaire d'institutions défaillantes, pour Mirabeau, l'asservissement de la nation et la corruption de l'espace public s'expliquent par l'oubli. Oubli contraint car le pouvoir absolu, en se concevant comme le seul aboutissement de tout destin collectif, s'est approprié le passé et il en a imposé la récriture en termes d'immobilisme et de continuité pour fonder sa propre légitimité. "Quant aux princes qui défendent avec acharnement leur autorité, ils ne sont redoutables que pour une nation qui ne connait ni ses droits ni ses forces ${ }^{64}$. $\gg$ Le despotisme ne peut se concevoir sans l'aliénation de l'espace de l'écriture de l'histoire, dont la place est occupée par un récit dévoyé qui constitue la seule justification de son existence : la tradition.

Pour Mirabeau, se pencher sur le passé n'est pas un geste innocent ; en pensant la mise en place du pouvoir absolutiste comme un processus évolutif, Mirabeau dénonce sa dimension temporelle, voire circonstancielle, et il refuse par là le double argument sur lequel s'était construite sa légitimation. S'interroger sur la mémoire de la nation constitue à proprement parler le premier geste de rébellion qui conduit au mûrissement d'une prise de conscience politique et qui permet de saisir les origines des maux qui intéressent la France contemporaine. Comme pour Mably et Boullainvilliers bien avant lui ${ }^{65}$, l'auteur de l'Essai sur le despotisme fait de l'histoire une arme critique et destructrice qui prend à rebours le récit royal : elle devient le premier espace de critique à l'institution monarchique dans la mesure où elle la dépouille de tous les arguments qui la rendaient 
intouchable car intemporelle. De ce point de vue, Mirabeau se montre bien fils d'un siècle soucieux de trouver dans le passé les raisons de l'effacement de la liberté politique en France et surtout l'héritier d'une vision de l'histoire typique des Lumières, qui faisait du changement le principal objet de son intérêt ${ }^{66}$.

Pourtant, tandis que ses prédécesseurs instruisaient un procès à la monarchie, pour ainsi dire, par voie interne, en s'opposant autour de la fameuse querelle Francs/Gaulois, Mirabeau enjambe consciemment les limites imposées par le cadre national en poursuivant sa réflexion par une mise en perspective de l'absolutisme français avec les événements du XVII ${ }^{e}$ siècle anglais. Ceux-ci sont considérés comme le moment culminant d'un processus spéculaire et contraire à l'histoire de France, où une nation a redécouvert et conquis la liberté politique et civile, même si elle l'a fait d'une manière contradictoire et provisoire. Dans son discours introductif à l'Histoire d'Angleterre de Macaulay, Mirabeau précise son point de vue :

Notre objet principal sera de montrer avec clarté l'influence que le gouvernement a toujours exercée sur les mœurs, et par conséquent sur la prospérité des Anglais. [...] Nous [montrerons] quels moyens, quelles ressources ils pouvaient, ils devaient employer pour arriver à cette liberté politique et civile, sans laquelle il n'est, pour aucune nation, ni paix, ni gloire, ni bonheur.

Mirabeau, en reprenant pleinement l'interprétation des faits proposée par Macaulay, relit l'affrontement du siècle précédent dans des termes moraux, le transformant en une lutte antinomique entre deux acteurs porteurs de solutions politiques opposées. D'un côté, le patriote, défenseur de «l'esprit public» qui aurait été légitimé dans sa lutte par l'universalité de son message et par sa capacité à défendre les intérêts de la nation entière, de l'autre le partisan de l'absolutisme qui n'était rien autre que le reflet des «intérêts particuliers" et de l'ignorance. Placée devant l'alternative de perdre définitivement la liberté sous le joug despotique, la nation a su se réapproprier ses propres droits et faire triompher les vertus collectives car, consciente de ses propres droits, elle l'a voulu. L'expérience anglaise dans sa globalité permet ainsi de développer un discours sur le devenir historique, dans le champ du politique en le considérant comme fruit d'un acte de volonté collective. Autrement dit, elle rend possible de concevoir la révolution comme moyen de régénération, qui découle d'une prise de conscience collective ${ }^{67}$.

Ainsi l'événement anglais peut être dépouillé de ses spécificités pour acquérir une dimension pédagogique en devenant un exemple de résistance, de "révolte» pour reprendre les termes du tribun, d'un peuple qui a su briser le poids de la tradition. En ce sens la guerre civile cesse d'être l'histoire de la nation anglaise pour devenir celle plus générale, et donc transnationale, des «vicissitudes de la liberté ». Par conséquent, les histoires de France et d'Angleterre peuvent être écrites et même pensées de manière autonome, mais, en lisant les vicissitudes de l'une, il est possible de penser la problématique de la liberté de l'autre et en suggérer ainsi un possible changement. Comme Mirabeau l'écrit lui-même, sans doute la connaissance des périodes anciennes est nécessaire aux nations pour faire chérir les formes de gouvernement actuelles, mais davantage encore, elle doit révéler :

Le contraste présent dans ces institutions mêmes, à côté des merveilles que pourrait produire la raison publique, armée de tous ses moyens, puisqu'il est vrai que le bien s'est quelquefois opéré sans autre appui qu'un peu de sagesse et de prévoyance $^{68}$. 
Au-delà des circonstances qui peuvent avoir déterminé son cours, la guerre civile anglaise devient la meilleure preuve d'une extranéité fondamentale de l'absolutisme, de son caractère factice et donc illégitime, lorsque la nation sait reconnaitre ses véritables intérêts. Cette interprétation de l'événement historique, qui par ailleurs renforce sa critique à toute forme de despotisme monarchique, conduit Mirabeau à justifier pleinement le régicide en tant qu'acte nécessaire, non pour réinventer le pacte social mais pour reconstruire un équilibre politique. Pour celui qui sera le conseiller inécouté de la couronne à partir de 1790, l'exécution de Charles $\mathrm{I}^{\mathrm{er}}$ est la punition méritée d'un roi oppresseur qui voulait réduire une nation à l'esclavage. Par-là, Mirabeau dépouille le geste de ses implications républicaines, ou du moins les amoindrit, mais il affirme clairement le droit souverain de la nation à juger ses gouvernants.

Quoi! Il ne faut point détester les oppresseurs? Mais lorsque la plus sévère et la plus exigeante des religions ordonna le pardon des injures privées, elle prescrivit le châtiment public de ces monstres qui vexent et déshonorent des nations entières ${ }^{69}$. Cette interprétation de l'événement permet enfin de saisir la valeur politique de la traduction qui se réintègre de façon cohérente avec le projet de Mirabeau favorable à l'établissement d'une monarchie patriote en France $^{70}$. Ce qui l'intéresse dans la Révolution anglaise sont moins ses issues républicaines ou son déroulement concret, Mirabeau évite soigneusement de citer les unes et l'autre dans le long discours préliminaire $^{71}$, que son importance en tant que précédent comme moment fondateur d'une tradition révolutionnaire. Mirabeau relit l'expérience du Commonwealth et les luttes qui les précédent au filtre d'un républicanisme classique, qui par ailleurs s'accorde parfaitement avec l'interprétation commonwealthmen de l'époque, en la considérant comme un affrontement entre deux entités morales. Selon cette perspective, l'exemple républicain est considéré et réintégré à l'intérieur d'un discours patriotique construit par antithèse au pouvoir corrupteur et dévoyé du despotisme, qu'il soit exercé par un roi ou par un tyran. Il offre une base historique pour créer ce que Baker a défini, «an oppositional language rather than as a prescriptive model, as offering a diagnognis rather than an ideal» et développer ainsi un «discourse of political will ${ }^{72}$.» En ce sens, la problématique et les choix institutionnels proprement dits demeurent secondaires. Sur ce point, sans doute, il modifie plutôt que reprendre les positions de Macaulay qui considérait les deux aspects liées.

Tout comme l'avait fait Brissot, Mirabeau plaide pour une connaissance de l'histoire d'Angleterre, parce qu'elle prouve que la liberté politique n'est ni acquise ni héritée du passé. Exactement comme son contraire le despotisme, elle est le produit, toujours en évolution, d'un parcours pluriséculaire dont les luttes politiques du XVII ${ }^{e}$ siècle ont été l'un des moments culminants. Ainsi dans les intentions de Mirabeau, les deux histoires nationales se croisent et, par un parfait jeu de miroir, elles rendent compte, en dépit de leurs issues profondément différentes, de la même tension politique : l'opposition qui existe entre les droits de la nation et la tentation despotique de la couronne.

Plus précisément, l'histoire du XVII siècle anglais occupe une place décisive et même fondatrice dans la pensée politique de Mirabeau, parce qu'elle constitue la base empirique complémentaire à l'histoire de France. La première lui permet de valider, de prouver en sens inverse les intuitions que la seconde lui avait inspirées. Promouvoir la traduction de l'histoire de la guerre civile anglaise au final n'implique pas pour Mirabeau de penser ou suggérer une destinée républicaine pour la France, elle signifie plutôt d'attribuer au passé une fonction dynamique dans le présent et légitimer un discours de régénération anti- 
absolutiste contre la tradition. L'exemple du Commonwealth génère une conscience de la liberté et permet de penser la rupture ${ }^{73}$.

Qu'on respecte les anciennes institutions, quand elles n'ont rien de malfaisant; mais lorsqu'elles sont fatales pourquoi ne pas les proscrire? Et si c'était un mérite que l'ancienneté, comment l'erreur pourrait-elle disputer cet avantage à l'éternelle vérité ? Comment ne pas sentir que le dernier des hommes peut exercer la place de visir, tandis qu'il faut allier tous les dons de la nature et de l'art pour préparer, pour mûrir une révolution, pour naturaliser la liberté dans des âmes accoutumées à

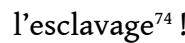

Envisageant la connaissance historique en tant qu'instrument pour développer une conscience commune à la nation qui est à la fois le préalable à l'action révolutionnaire et le socle de la future monarchie patriote, Mirabeau postule une responsabilité civique pour l'historien qui assume un rôle clé décisif dans l'espace du politique. Le futur Constituant se pense comme un véritable aiguilleur des consciences sans lequel tout changement est impossible, car si des institutions légitimes permettent de conserver la liberté, elles ne peuvent se soutenir que si les administrés sont assez mûrs pour les accepter ${ }^{75}$. Son œuvre de transmission assume pleinement une valeur pédagogique : en dévoilant les "illusions » qui entourent le passé, et "souvent le ternissent », elle détruit le "préjugé » et elle devient un moyen d'éducation collective qui accélère le progrès ${ }^{76}$. L'impartialité de l'historien ne se mesure pas ainsi seulement dans sa capacité de décrire l'événement tel qui s'est passé, mais aussi et surtout dans celle de l'interpréter, de le mettre à disposition pour perfectionner la société à laquelle il appartient. Sa tâche n'est donc pas de présenter un «tableau» mais de construire une narration capable d'exprimer un jugement sur la réalité.

41 C'est ici que, selon Mirabeau, au-delà de la proximité historiographique qui le lie à l'historienne anglaise, se fonde véritablement la force de l'histoire de Macaulay et sa supériorité sur celle de Hume, dont l'œuvre est critiquée à plusieurs reprises tout au long de son discours introductif et constituée en véritable repoussoir. L'historienne républicaine «a toujours eu en vue le bien du peuple; ce bien, l'objet unique de tout gouvernement; ce bien auquel l'histoire doit ramener toutes les actions des hommes pour les juger sainement ${ }^{77}$.» Elle incarne en quelques sortes le modèle ou l'archétype même de l'historien-magistrat capable de transformer la discipline de clio dans un tribunal impartial capable de permettre la régénération collective de la nation.

o Hume ! [...] Il ne suffisait pas d'être [...], le rapporteur impartial de tous les faits, de toutes les opinions. Il fallait reculer l'enceinte dans laquelle tes compatriotes ont circonscrit la liberté civile et politique, [...] tonner contre les oppresseurs; et cette illustre Macaulay, dont les talents, quoique distingués, sont incontestablement inférieurs au tien, ne t'eût ni enlevé ni même disputé, la palme de l'histoire ${ }^{78}$.

Pour conclure, à travers le travail effectué sur le texte de Macaulay, Mirabeau non seulement se révèle un penseur capable d'interroger deux traditions nationales autour de la même question de la liberté, mais il est aussi une figure de passage entre deux conceptions différentes de l'usage politique de l'histoire: derrière la vieille idée d'un tribunal impartial pour juger le despotisme avance déjà le problème de l'éducation de la nation dans un espace révolutionnaire. 


\section{Traduire Macaulay pour former une identité collective} nom de la tradition, reprise par Brissot et par Mirabeau pour développer un discours sur la rupture, la signification de l'Histoire d'Angleterre change sous l'emprise des circonstances à peine quelques mois plus tard. Le despotisme terrassé, elle devient une prise de conscience des problèmes connexes à la construction de l'ordre révolutionnaire et à la sauvegarde de la liberté. C'est au prisme de cette urgence qu'il faut lire la préface de Charles-Philippe Guiraudet qui accompagne la sortie du troisième volume de l'histoire, publié à la fin de l'été 1791 .

de limiter a sa tâche de tracteur ${ }^{79}$, l'ancienne plume de Mirabeau prouve le besoin de justifier politiquement la publication de ce travail que les péripéties contemporaines rendaient d'une actualité brulante. Surtout, il veut mettre en garde son public contre le risque d'un dérapage de la Révolution qu'il estime imminent et, pour cela, il n'hésite pas à faire de l'histoire républicaine de Macaulay un plaidoyer en faveur de la monarchie des Bourbons. Paradoxe certes, mais paradoxe intéressant car son auteur s'efforce de le justifier au nom des mêmes principes de liberté et à travers les mêmes exemples historiques que Brissot et Girey-Dupré avaient utilisé à peine quelques semaines auparavant. Ce régime républicain canonisé comme modèle de vertu et de valeur, selon l'ancien collaborateur de Mirabeau, est d'abord et avant tout un régime qui a échoué à l'épreuve des faits, car, après avoir exécuté un roi, l'Angleterre s'est retrouvée dominée par un tyran. Est-ce réellement le destin souhaité par les nouveaux "nivelleurs» français?

Hommes pervers, ou insensés, ignorants, ou légers qui ne connaissez de la Révolution anglaise, que ce qui l'a souillée, je veux dire le supplice de Charles! [...] Apprenez que l'époque de sa mort fut celle du dernier degré d'avilissement où une nation pût être plongée, et prépara le despotisme de l'usurpateur sous lequel elle eût à servir ${ }^{80}$ !

Les vicissitudes du Commonwealth témoigneraient de l'impossibilité d'établir la démocratie et la république dans un grand état : les deux ne seraient rien d'autre qu'une étape préalable au Chaos et à la servitude. Compte tenu du contexte, il est évident que Guiraudet s'appuie sur le passé pour se faire le relai du discours que les meneurs Feuillants avaient répété avec bien plus de force lors des débats contemporains à l'Assemblée nationale. En ce sens, l'exploitation militante et politicienne que Guiraudet fait de l'histoire de Macaulay est d'autant plus originale qu'elle déforme le contenu du texte sur lequel il s'appuie.

Tout l'intérêt de l'intervention de Guiraudet réside ailleurs, dans l'utilisation que la traducteur fait de la guerre civile pour penser l'accélération du temps en reconstruisant parallèlement un ordre à l'intérieur de l'aporie qui parcourt le moment révolutionnaire dont il est à la fois témoin et protagoniste.

Face au délitement du front patriote qui avait mené la Révolution jusqu'alors, événement qu'il considère comme la meilleure preuve du fait que la rupture historique appelée de tous les vœux par Mirabeau a précédé la régénération morale dont il aurait dû être l'effet, Guiraudet attribue au passé anglais une fonction d'avertissement destiné aux révolutionnaires dans leur ensemble sur la dynamique de l'événement auquel ils

La Révolution française, 5 | 2013 
participent. Il constitue un avertissement sur le fait que la Révolution, elle-aussi, a un cours et il peut être ruineux en retardant l'affirmation des principes qui l'ont inspirée. Les circonstances ont leur poids et elle n'est pas seulement l'œuvre de la volonté. Il ne suffit pas de proclamer la rupture, il faut encore l'achever. Pour édifier la liberté, il est nécessaire de la penser comme une entité historique et prendre conscience de sa fragilité. Pour reprendre l'expression même du traducteur, la véritable langue des nations qui cherchent à remodeler leurs institutions doit être le passé et non une grammaire abstraite de la société. L'histoire malheureuse du Commonwealth doit constituer la véritable base sur laquelle construire à nouveau l'unité des patriotes.

L'histoire de la révolution d'Angleterre a été écrite, sans doute, mais pour pouvoir en appliquer les résultats d'une manière éclairée à notre position actuelle [...] il faut que les faits soient connus du plus grand nombre [...]; il importe que les lumières qu'ils peuvent répandre puissent être aperçues de tous les partis, [...] et que chacun en comparant les deux révolutions, puisse en saisir les rapports et les différences ${ }^{81}$.

S'il est un avertissement pour les révolutionnaires eux-mêmes, l'ouvrage de Macaulay recèle un enseignement encore plus important pour les opposants de la Révolution. Selon Guiraudet, les émigrés auraient dû faire de l'Histoire d'Angleterre leur livre de chevet et apprendre que « tout intermédiaire entre le peuple et le roi doit être repoussé quand il ne peut plus servir ou se faire craindre; que tous corps qui, sans avoir une consistance politique, se trouve placé entre ces deux grands arcs-boutants politiques, doit périr.» Quant au roi, il serait tenu de méditer sur le sort de son prédécesseur pour apprendre qu'il n'a reçu sa place que pour en « rendre compte à la nation entière... Que le passé vous [Louis XVI] éclaire sur l'avenir ${ }^{82}$.» L'histoire de la guerre civile se transforme en une exhortation à reconnaitre l'inéluctabilité du changement historique. Elle sert à rendre évident le lien de nécessité qui a conduit la transformation des anciennes monarchies absolues en royaumes fondés sur la volonté populaire. Si la Révolution peut encore échouer, l'Ancien régime en revanche ne pourra plus jamais revenir. Le souvenir des luttes du XVII ${ }^{e}$ siècle devient alors le meilleur démenti de toute prétention à effacer la rupture de 1789 .

La préface de Guiraudet transforme l'Histoire d'Angleterre de Macaulay en un cours de politique pour comprendre la portée globale de la rupture qui s'est opérée en 1789 . Ni repoussoir ni modèle à suivre ${ }^{83}$, elle devient plutôt un outil épistémologique pour comprendre le fait révolutionnaire et lui attribue au même temps une fonction pédagogique pour former une nouvelle identité collective.

Tandis que Mirabeau pouvait imaginer la révolution en termes antinomiques comme une lutte contre le despotisme de la part d'une nation unie et fraternelle, les mots de Guiraudet sont, en revanche, le témoignage des craintes d'une élite patriote désabusée par les rebondissements de l'année 1791. Il infléchit sans doute la confiance qui était celle de son ami par une identité suggérée entre renversement du despotisme et progrès, mais il ne remet pas en cause une histoire institutrice de la liberté politique. Bien au contraire, l'importance de la traduction de Macaulay en sort accrue parce qu'elle constitue un désenchantement qui, en détruisant le mythe d'un recommencement absolu, inscrit la Révolution de 1789 dans une parcours historique. Elle éclaire la dynamique de l'histoire universelle dans sa globalité, fondant ainsi sa légitimité. À Guiraudet de conclure :

Ce sont des exemples, des faits, qui peuvent seuls servir à l'éducation des peuples, comme à celle des individus. [...] L'individu peuple ne reçoit, comme chacun des membres qui le composent, des leçons, que de l'expérience, des conseils utiles, que de ses erreurs, des instructions, que du malheur et du temps. Des faits et des 
exemples analogues, sont les seuls moyens d'abréger ces cruels moments d'épreuve

[...] voilà désormais l'utile et sublime emploi de l'histoire ${ }^{84}$.

51 politique dont il espérait voir la stabilisation. De la même façon, se termine brutalement l'entreprise éditoriale qui, à l'origine prévoyait la publication intégrale des huit volumes écrits par Macaulay, auxquels aurait dû s'ajouter une série d'annexes. L'éditeur avait prévu de conclure l'ouvrage par « un tableau comparé » des « deux révolutions, dont la première a dû éclairer et produire la seconde, qui à son tour en produira tant d'autres ${ }^{85}$. » A en croire l'éditeur, l'ensemble des textes était déjà prêt et traduit lors de la sortie du premier volume mais le manque de moyens financiers avait imposé une publication échelonnée dans le temps, dans l'espoir que les recettes des tomes initiaux fussent en mesure de financer les frais des suivants. Ce mécanisme, assez courant à l'époque, laisse supposer que l'ouvrage a rencontré un succès initial comme l'attestent aussi les critiques positives des journaux et surtout l'introduction au troisième volume, publié dans la foulée des deux premiers ${ }^{86}$. En effet, tout porte à croire que l'arrêt impromptu de l'Histoire d'Angleterre au cinquième volume s'explique davantage par le contexte de crise de l'été 1792 et par l'écroulement du marché du livre qui s'en suit, plutôt qu'à cause d'un désintérêt spécifique pour le livre en question. Ce n'est pas un hasard d'ailleurs si le quatrième et le cinquième tome sont les derniers ouvrages imprimés par l'éditeur Gattey avant sa faillite en octobre ${ }^{87}$. Le moment de lire une histoire républicaine était passé, celui de la faire était advenu : lorsque le cinquième tome, qui s'achevait sur l'exécution d'un roi venait d'être mis en vente, le procès d'un autre allait s'ouvrir, au nom des mêmes valeurs républicaines que Catharine Macaulay avait voulu léguer à la postérité. Le passage de témoin était complet.

\section{Conclusions}

52 À travers la mise en perspective des interventions qui se sont succédées autour de l' Histoire d'Angleterre de Catherine Macaulay, l'opportunité est faite de montrer comment la publication de cet ouvrage trouve sa place à l'intérieur d'un discours sur l'histoire cohérente qui explique, de manière plus générale, la résurgence du souvenir de la Révolution anglaise lors de la crise du siècle suivant de l'autre côté de la Manche.

Surtout, par le biais de cette traduction, explorer la relation qui lie dynamique révolutionnaire et évolution des représentations du passé devient possible. Si la rupture de 1789 favorise l'affirmation d'une nouvelle manière de travailler son objet d'études et même des nouveaux critères d'écriture, elle produit aussi un profond changement dans l'usage public du passé. La Révolution favorise une canonisation inédite du passé qui acquiert une portée politique dans la mesure où elle permet de construire une conscience du changement à partir de laquelle peut être pensée et réalisée la nouvelle rupture ${ }^{88}$. De contre-pouvoir capable de révéler le mensonge monarchique et briser son monopole interprétatif sur le passé, l'histoire acquiert une fonction nouvelle car elle censée fonder ou du moins contribue à fonder une identité collective à deux égards.

refence au passé instaure la rupture et, à travers la notion de progrès, elle la rend irréversible en la légitimant. Le passé n'est plus convoqué en tant qu'auctoritas capable d'influencer l'élaboration d'une ligne politique. Si cet aspect est encore en partie présent comme le démontre la préface de Guiraudet, ce n'est pas là l'essentiel. Les hommes de 1789, héritiers des philosophes, ont intériorisé le dépassement de l'histoire magistrae vitae

La Révolution française, 5 | 2013 
opérée tout au long du siècle par l'historiographie des Lumières : ils sont convaincus que la Révolution anglaise est irréductiblement différente par rapport à la situation française. Alors, non seulement, comme l'écrit Pierre Serna à propos de l'historiographie révolutionnaire immédiate, le récit du passé «joue [...] le rôle de relance du processus décrit, en suggérant ce qu'il reste à faire, par rapport ce qui a été fait dans le passé ; ce qui risque de se produire, en fonction, de ce qui est survenu, jadis ${ }^{89}$ "; sur un plan plus général il permet de développer un discours global sur la révolution en tant qu'événement, de la conceptualiser en tant qu'objet politique. Le passé anglais permet d'attribuer une signification propre à la rupture, au milieu et en dépit des rebondissements auxquels est soumis tout événement politique majeur. En ce sens, l'histoire de l'Angleterre constitue une référence dans la mesure où elle dévoile les règles d'un devenir historique dont les événements actuels seraient l'aboutissement et non pas sa disqualifiation. Pour reprendre les termes de Jean-Marie Goulemot, les écrits de Macaulay, d'abord cités pour penser et favoriser la rupture, conçue en termes moraux, deviennent par la suite un outil intellectuel qui favorise «la mémorisation de la Révolution et [...] son inscription dans une histoire où elle trouverait sa place parce qu'on lirait dans ce qui la précède les causes même de sa venue ${ }^{90}$. " En dernière analyse, la réflexion sur l'histoire du XVII siècle fonde un nouveau cadre qui rend possible l'action collective en donnant la possibilité de se replacer dans un univers temporel qui garde par ailleurs des implications morales. Pour renverser le fameux constat de Marx, le regard sur le passé ne leurre pas les révolutionnaires mais leur permet de se saisir du contenu de leur propre action.

Deuxième aspect, le souvenir du passé est utilisé pour construire un imaginaire commun capable de récréer la nouvelle communauté nationale autour des valeurs dotées d'une signification métahistorique. Si l'histoire de France était le récit d'une progressive décadence dont le triomphe de l'absolutisme représentait le trait unificateur et saillant, les révolutionnaires sont obligés de chercher aussi et surtout ailleurs des éléments sur lesquels fonder la légitimité de la nation régénérée. Si Pierre Nora constate à juste titre qu'« en dressant rapidement le spectre rétrospectif de "l'ancien régime", dont la disparition en tant que tel devenait la condition première de son avènement, la nation souveraine s'est privée au départ et par principe des huit siècles de continuité temporelle qui constituaient sa vraie légitimité ${ }^{91}$ ", on comprend alors que l'histoire de la guerre civile anglaise devienne une tentative d'ancrer, de donner des repères à la révolution actuelle dans un temps historique. Autrement dit, le XVII ${ }^{e}$ siècle anglais contribue à créer un champ d'expérience commun où peuvent se reconnaitre tous les patriotes qui sont en train d'accomplir la Révolution de 1789, il devient un espace de mémoire partagée sur lequel construire une nouvelle culture politique alternative à l'absolutisme monarchique. Ceci explique pourquoi, sans jamais atteindre la popularité de l'Histoire d'Angleterre de David Hume ${ }^{92}$, l'ouvrage de Macaulay s'affirme progressivement comme le texte de référence pour ceux qui contestaient le message d'une histoire contrainte de refonder le pacte social par des principes puisés dans la tradition ancienne. Le souvenir des révolutions du passé permet notamment de reconstituer l'unité mémorielle de la nation régénérée, épurée de ses opposants, projeté vers un projet futur. En tissant des liens nouveaux, l'histoire de la liberté exclue et par là, forge une identité collective.

En ouverture de son magnifique livre dédié à la mémoire de la guerre civile anglaise, Roundhead Reputations, Blair Worden écrit que «the wars, which tore the nations apart, have been fought again in the heart of posterity. The clash of Cavalier and Roundhead has 
never gone away ${ }^{93}$.» Cent-cinquante ans plus tard, de l'autre côté de la Manche, le bruit de la guerre civile résonne plus fort que jamais en marquant un chemin de conquête de la liberté.

\section{NOTES}

1. À ce propos, voir le célèbre ouvrage de Louis-Thomas LAVICOMTERIE, Les crimes des rois de France depuis Clovis jusqu'à Louis XVI, Paris, Petit, 1791, p.16; sur le rapport entre histoire et républicanisme Patrice GUENIFFEY, «Les républicains avant la République », dans François FURET et Mona ozouf (dir.), Le siècle de l'avènement républicain, Paris, Gallimard, 1993, p. 197-224. En effet, les rois lisaient attentivement l'histoire de leurs prédécesseurs. Par exemple : sur Charles $\mathrm{I}^{\mathrm{er}}$ en tant que lecteur de Davila : Paulina Kewes, « History and Its Uses », dans Paulina KEWES (dir.), The uses of history in Early Modern England, San Marino, California, Huntington library, 2006, p. 19-21 ; Sur Louis XVI lecteur de Hume: Laurence Louis BONGIE, David Hume, Prophet of the Counterrevolution, Oxford, Clarendon Press, 1965, p. 120-130 et passim.

2. Jacques-Pierre BRISSOT, Discours du 10 juillet 1791 à l'assemblée des Amis de la Constitution, le 10 juillet 1791, ou Tableau frappant de la situation actuelle des puissances de l'Europe, s.l., s.d. Sur les rapports entre Brissot et la tradition républicaine anglaise, voir aussi dans ce numéro Pierre SERNA, Le pari politique de Brissot ou lorsque le Patriote Français, l'Abolitionniste Anglais et le Citoyen Américain sont unis en une seule figure de la liberté républicaine.

3. Joseph Marie GIREY-DUPRÉ, Opinion sur la question de savoir quelle sera la conduite de l'Assemblée nationale, à l'égard du roi, Paris, Imprimerie nationale, 1791, p. 8.

4. Pierre SERNA, «1799, Le retour du refoulé ou l'histoire de la révolution anglaise à l'ordre du jour de la crise du Directoire ", dans Philippe BOURDIN (dir.), La Révolution, 1789-1871 : écriture d'une histoire immédiate, Clermont-Ferrand, Presses Universitaires, 2008, p. 213-240, et Olivier LUTAUD, Des révolutions d'Angleterre à la Révolution française : le tyrannicide et « Killing no murder, Cromwell, Athalie, Bonaparte ", essai de littérature politique comparée, La Haye, M. Nijhoff, 1973, p. 3.

5. Pour les exceptions, outre les références de la note qui précède, voir aussi Olivier LUTAUD, « Des révolutions d'Angleterre à la Révolution française : l'exemple de la liberté de presse ou comment Milton " ouvrit » les États généraux », dans Christian CROISILLE et Jean EHRARD (dir.), La Légende de la Révolution, Clermont-Ferrand, Centre de recherches révolutionnaires et romantiques, 1988, p. 115-24; Olivier LUTAUD, «Emprunts de la Révolution française à la première révolution anglaise. De Stuart à Capet, de Cromwell à Bonaparte", dans Revue d'histoire moderne et contemporaine, t. 37e, n 4 (Oct. - Dec., 1990), p. 589-607 et surtout Rachel HAMMERSLEY, The English republican tradition and eighteenth-century France : between the ancients and the moderns, Manchester, University Press, 2010.

6. Elles auraient été opposées, car leur dialectique structurait deux modèles politiques inversés. Par exemple, héritier en ce sens de toute l'historiographie britannique du XIX ${ }^{\mathrm{e}}$ siècle, Philip Antony BROWN, The French Révolution in English History, London, Lockwood and sons, 1918. Un bon résumé de la question : Edward ROYLE, Revolutionary Britannia? Reflection on the treat of revolution in Britain, 1789-1848, Manchester, University Press, 2000, p. 1-10. Sur la fonction de l'ennemi français dans la construction de l'idée de nation en Angleterre au XVIII ${ }^{\mathrm{e}}$ siècle, entre autres : Linda COLLEY, Britons : Forging the Nation, New Haven (Conn.), University Press, 2005. Ce constat n'empêche pas 
que, au-delà du profond renouveau de l'histoire transnationale des dernières années, il ait toujours existé un intérêt scientifique pour les échanges et les rapports entre les deux nations au XVIII ${ }^{\mathrm{e}}$ siècle, dès l'époque de François Guizot. Pour les derniers travaux: Christophe CHARLE, Julien VINCENT, Jay WINTER (dir.), Anglo-French attitudes: comparisons and transfers between English and French intellectuals since the eighteenth century, Manchester, University Press, 2007 et Ann THOMsON, Simon BURRows, Edmond DZIEMBOWSKI (dir.), Cultural transfers: France and Britain in the long eighteenth century, Oxford, Voltaire foundation, 2010.

7. Edmund BURKE, Réflexions sur la Révolution de France, Paris, Pluriel, 2011 [1790] et Edmund BURKE, Lettre à un membre de l'Assemblée nationale sur la Révolution française et Rousseau, Paris, Ed. Mille et une nuits, 2012 [1791].

8. Mona ozouf, «La Révolution française et l'aléatoire : l'exemple de Varennes ", dans Mona OzoUf, L’homme régénéré : essais sur la Révolution Française, Paris, Gallimard, 1989, p. 92, 116-156.

9. Reinhart KOSELLECK, Le Futur Passé, Paris, Ed. EHESS, 1990, p. 71-76 et François HARTOG, Régimes d'historicité, présentisme et expérience, Paris, Ed. du Seuil, 2003.

10. Jean-Marie Goulemot, « Notes sur la mémorisation de la Révolution française à la fin du dixhuitième siècle. ", dans Romantisme, $\mathrm{n}^{\circ}$ 51, 1986, p. 17-22 ; Louis-Thomas LAVICOMTERIE, Du peuple et des rois, op. cit., p. 10 ; Adrien BARNAVE (Patrice GUENIFFEY, dir.), De la révolution et de la constitution, Grenoble, Presses Universitaires Grenoble, 1988 ; Jean-Paul RABAUD SAINT ETIENNE, Précis historique de la Révolution française, Paris, Didot, 1792.

11. Keith Michael BAKER, Au tribunal de l'opinion, Paris, Payot, 1993, p. 84.

12. Louis-Thomas LAVICOMTERIE, Du peuple et des rois, op. cit., p. 52.

13. Rachel HAMMERSLEY, The English republican tradition, op. cit.

14. Catharine MACAULAY, The History of England from the Accession of James I to that of the Brunswick Line, London, J. Nourse (C. Dilly), 1763-1783, 8 vol. Il sera traduit sous le titre de Catharine MACAULAY, Histoire d'Angleterre, depuis l'avènement de Jacques I jusqu'à la révolution, traduite en français et augmentée d'un discours préliminaire, contenant un précis de l'histoire d'Angleterre jusqu'à l'avènement de Jacques I, et enrichie de notes par Mirabeau, Paris, Gattey, 1791-1792, 5 vol.

15. Réimpression de l'ancien Moniteur Universel, Paris, 1847, t. XI, nº 45, 14 Février 1792, p. 376 ; Ivi., t. X, n² 282, 9 octobre 1791, p. 62. Italique réalisées par l'auteur.

16. Pour un cadre globale des traductions lors de la Révolution: Charles Alfred ROCHEDIEU, Bibliography of French Translations of English Works, 1700-1800, Chicago, University Press, 1948. En revanche, un tableau général des écrits français qui font référence à la Guerre Civile anglaise reste encore à élaborer.

17. Rachel HAMMERSLEY, The English republican tradition, op. cit., p. 175-180 ; François QUASTANA, La pensée politique de Mirabeau, 1771-1789: "républicanisme classique" et régénération de la monarchie, Aixen-Provence, Presses Universitaires, 2007, p. 126-127.

18. Cette popularité politique du système constitutionnel anglais est due aux travaux de Voltaire et Montesquieu, qui en effet sont profondément influencés par les positions whig. voltaire, Lettres philosophiques, Paris, Garnier, 2010 [1734] et Charles-Louis Secondat de MONTESQUieu, De l'esprit des lois, Paris, Flammarion, 2008 [1748] ; Joseph DEDIEU, Montesquieu et la tradition politique anglaise en France : les sources anglaises de l'esprit de lois, Paris, J. Gabalda, 1911. Edmond DZIEMBOWSKI , «The English political model in eighteenth-century France », dans Historical Research, LXXIV, 2001, p. 156-157.

19. Entre autres: Philip HICKS, Neoclassical history and English Culture from Clarendon to Hume, London, Palgrave, 1996; Laird OKIE, Augustan Historical Writing, Histories of England in the English Enlightenment, Lanham, University Press of America, 1991; Roger Charles RICHARDSON, The debate on the English revolution, Manchester, University Press, 1998, p. 40-64.

20. David HUME, The history of England, from the invasion of Julius Coesar to the Revolution in 1688, London, Millar, 1754-1762, 6 vol. 
21. Cette interprétation est développée dans le vol. $\mathrm{V}$ de l'ouvrage complète, qui avait été le premier à être publié. David HUME, The history of England, under the house of Stuart, London, Millar, 1754. C'est le volume le plus important du point de vu historiographique et politique. À ce propos: Victor G. WEXLER, David Hume and the History of England, Philadelphia, American Philosophical Society, 1979, p. 2-42; Mark SALBER PHILLIPS et Dale SMITH, «Canonization and Critique: Hume's Reputation as a Historian ", dans Peter JONES, The reception of David Hume in Europe, London, Thoemmes Continuum, 2005 p. 299-314; Mark SALBER PHILLIPS, "The most Illustrious Philosopher and Historian of the Age: Hume's History of England », dans Elizabeth SCHIMDT RADCLIFFE, A companion of Hume, Oxford, Blackwell, 2008.

22. Richard D. DEES, "One of the Finest and the Most Subtile invention: Hume on the Governement ", dans E. SCHIMDT RADCLIFFE, A companion of Hume, op. cit., p. 388-404.

23. David HUME, of the coalition of Parties, 1758, cité par Victor G. WEXLER, David Hume and the History of England, op. cit., p. 42 et aussi voir l'échange épistolaire du 29 mars 1764 avec Catharine Macaulay cité dans The European Magazine and London Review, november 1783, vol. 56, p. 332-334.

24. Victor G. WEXLER, David Hume and the History of England, op.cit. et Duncan FORBES, "Introduction », dans David HuME, History of Great Britain: the Reigns of James I and Charles I, Harmondsworth, Penguin, 1970, p. 5-15.

25. John Greville Agard Рососк, « Catharine Macaulay: patriot historian », dans Hilda SMITH (dir.), Women writers and the early modern British political tradition, Cambridge, University Press, 1998, p. 257.

26. La publication de la version originale du livre de Macaulay commence en 1763 pour se terminer vingt ans après, en 1783. Cependant, treize ans de vide séparent les cinq premiers volumes dédiés à la guerre civile anglaise, les plus importants, des trois derniers consacrés à la deuxième partie du XVII ${ }^{\mathrm{e}}$ siècle et à la Restauration. Nombreux travaux ont été dédiés à la personnalité et à l'œuvre de Macaulay, longtemps sous-évalué. Je me limite ici à citer les travaux utilisés à cette occasion: Bridget HILL, Christopher HILL, «Catharine Macaulay and the Seventeenth Century ", The Welsh History Review, 3, 1967, p. 381-402; Bridget HILL, The Republican Virago, Oxford, Clarendon Press, 1992, p. 24-51; Claire GILBRIDE FOX, "Catharine Macaulay, An Eighteenth- Century Clio», dans Winterthur Portfolio, vol. 4 (1968), p. 129-142; Lynne E. WiTHEY, "Catharine Macaulay and the Uses of History: Ancient Rights, Perfectionism, and Propaganda ", dans Journal of British Studies, vol. 16, nº 1 (Autumn, 1976), p. 59-83.

27. Hume lui-même le reconnaît lors d'un échange épistolaire avec l'auteure, "we differ less in facts, than in our interpretation and construction of them ", lettre à Macaulay du 29 mars 1764 dans, The European Magazine and London Review, November 1783, vol. 56, p. 332-334. Karen O'Brien préfère, en revanche, souligner la continuité entre Hume et Macaulay, en reconduisant cette dernière dans la cadre de l'historiographie des Lumières : Karen O'BRIEN, Women and Enlightenment in Eighteenth-Century Britain, Cambridge, 2009, p. 155-159.

28. Lynne E. WITHEY, « Catharine Macaulay and the Uses of History », op. cit., p. 69.

29. "This emphasis on individual morality lay at the heart of Macaulay's method of writing history. », Ivi, p. 72. Le corollaire méthodologique est que Macaulay considère l'histoire, conçue exclusivement du point de vue politique, comme un espace d'affrontement entre volontés et valeurs dont les régimes seraient la projection.

30. C'est l'auteure elle-même qui, dans les introductions à son ouvrage, souligne les rapports étroits entre écriture historique et engagement civique: « Animated with the love of liberty, and an enthusiastic regard to English patriotism, I ventured to take the pen in hand, with the intention of vindicating the insulted memoires of illustrious ancestors, [...].This, without any unconstitutional design, or any wild enthusiastic hope of being able to influence the minds of a nation in favor of a democratic form of government, who from the beginning of time have been under the rule of regal sway, and whose laws, manners, customs, and prejudices are ill adapted to 
a republic, is the grand aim of my writings. ", dans C. MACAULAY, The History of England, op. cit., vol. I, p. 7.

31. «History, in other words, told the story of the loss of the rights of man and also the progress toward realizing them again in a more perfect state.», dans Lynne E. WITHEY, "Catharine Macaulay and the Uses of History ", op. cit., p. 59.

32. Catharine maCAUlay, History of England, op. cit., vol. I, p. 10.

33. Ce qui explique toute l'ambivalence du jugement commonwealthmen sur la Constitution dans la deuxième moitié du siècle. Comme l'écrit Worden, « The very frequency of eighteenth-century praises of the English constitution hints at he unease behind them, at the awareness of the frailty and instability of the constitutional balance and of the post-Revolutionary political world that depended of it. ", Blair WORDEN, Roundhead Reputations, The English Civil War and the Passions of Posterity, London, Penguin, 2002, p. 162. Pour la vision de Macaulay sur la Glorieuse Révolution voir Catharine MACAULAY, History of England from the Revolution to the present time in a Series of Letters to a Friend, Bath, 1778, 2 vol.; J. G. A. Рососк, "Catharine Macaulay: patriot historian ", op. cit., p. 254-257; Lynne E. WITHEY, «Catharine Macaulay and the Uses of History», op. cit., p. 60-62 et Barbara BRANDON-SCHNORRENBERG, «An Opportunity missed: Catherine Macaulay on the Revolution of 1688 ", dans Studies in Eighteenth-Century Culture, n² 20, 1990, p. 231-240.

34. Catherine macaulay, The History of England, op. cit., vol. I, p. 16 et 20.

35. Barbara BRANDON-SCHNORRENBERG, «The Brood Hen of Faction: Mrs. Macaulay ad Radical politics, 1765-1775 », dans Albion, vol. 11, n 1 (Spring 1979), p. 33-45; Verner W. CRANE, « The Club of Honest Whigs: Friends of Science and Liberty », dans The William and Mary Quartely, Third Series, vol. 23, nº 2 (IV, 1966), p. 210-233; Lynne E. WITHEY, «Catharine Macaulay and the Uses of History ", op. cit., p. 60-62.

36. Pour un résumé des positions wilkities: Harry Thomas DICKINSON, Liberty and Property, London, Methuen, 1979, p. 195-220.

37. «In the years between 1760 and 1775 there can be no question that Catherine Macaulay was in the center of the radical cause in Great-Britain. Certainly she was accepted by the radical of the day as their primary theoretical spokesperson.", dans Barbara BRANDON-SCHNORRENBERG, "The Brood Hen of Faction... », op. cit., p. 44. Ce qui explique par ailleurs pourquoi Macaulay en cette période ne continue pas son histoire: son intérêt se concentre sur la politique contemporaine.

38. Catherine macaulay, History of England, op. cit., vol. I, p. 6. Sur la question Corse: Catharine MACAULAY Loose Remarks on certain position to be found in Mr Hobbe's "Philosophical rudiments of govenement and society" with a short sketch of a democratical form of government, in a letter to Signor Paoli, London, Johnston, 1767. Pour les rapports avec les insurgés americains: Catharine MACAULAY , An Adress to the People of England, Ireland, and Scotland on the Present Important Crisis Affairs, London, Johnston, 1775; Catherine MACAUlaY, History of England, op.cit., vol. VI, p. 1-6 et Carla H. Hay, "Catharine Macaulay and the American Revolution", dans Historian, t. 56, n 2 (1994, Winter), p. 301-316 Pour la polemique avec Burke, Catharine MACAULAY, Observations on the Reflections on the Right Hon. Edmund Burke, on the Revolution in France, London, C. Dilly, 1790.

39. Jacques-Pierre BRISSOT, Testament politique de l'Angleterre, Philadelphie, s.e. 1780. Pour l'évolution de l'image de l'Angleterre en France dans les années 1770-80: Gabriel BonNo, La Constitution Britannique devant l'opinion publique française de Montesquieu à Bonaparte, Paris, H. Champion, 1932, p. 133-190 ; Frances AсомB, Anglophobia in France, Durham, North Carolina, Duke University Press, 1950 ; Edmond DZIEMBOWSKI, « The English political model in eighteenth-century France ", op. cit., p. 168-171.

40. Jacques-Pierre BRISSOT, (Claude PERroud, dir.), Mémoires 1754-1793, Paris, Picard, 1911, t. 1, p. 347.

41. Ivi, p. 348. 
42. Brissot assume les changements qu'il opère à l'intérieur du texte. «La politique a bien changé, a fait un grand pas depuis la publication de cette Histoire [1764] et j'ai voulu la mettre au niveau de l'accroissement de cette science. Je voulais d'ailleurs être surtout utile au peuple.", dans Jacques-Pierre BRISSOT, Lettres philosophiques et politiques sur l'histoire de l'Angleterre depuis son origine jusqu'à nos jours, Londres et Paris, Regnault 1790 [1786], 2 vol, p. 23. La traduction de l'ouvrage serait de la femme de Brissot, Félicité, tandis que le futur conventionnel serait l'auteur de l'appareil critique et de la préface. Pour l'original, Olivier GoLDSMITH, An History of England, in a series of letters from a nobleman to his son, Londres, Newberry, 1764, 2 vol.

43. Ivi, p. 9-10.

44. Ivi, p. 14.

45. Ivi, p. 15. Brissot cite, en les attribuant à un auteur français, des extraits d'un autre ouvrage qui avait publié deux ans auparavant: le Journal du lycée de Londres. Pour ce qui concerne le passage dédié à Hume : Jacques-Pierre BRISSOT, Journal du lycée de Londres, Londres et Paris, Perisse le jeun, t. II, $\mathrm{n}^{\circ}$ 3, p. 162, 1785 ; pour celui de Macaulay Ivi, 1783, t. I, nº 1, p. 33, 1784. Une autre référence à The History of England qui remonte à la même période: Jacques-Pierre BRISSOT, Correspondance universelle sur ce qui intéresse le bonheur de l'homme et de la société, London, Cox, t. II, p. 54,1783 .

46. Brissot notamment reprend l'avant-propos de l'Essai sur les mœurs de Voltaire. VolTAIRE, Essai sur les mœurs et l'esprit des nations, depuis Charlemagne jusqu'à nos jours, Paris, Ed. Sociales, 1962 [1757] et Jacques-Pierre BRISSOT, Lettres philosophiques, op. cit., vol. I, p. 3-7.

47. Jacques-Pierre BRISSOT, Mémoires 1754-1793, op. cit., vol. I, p. 349. Italique réalisées par l'auteur.

48. Jacques-Pierre BRISSOT, Lettres philosophiques et politiques, op. cit., p. 14. Par la suite Macaulay et l'exemple de la Révolution anglaise resteront toujours présents dans l'esprit de Brissot. Je signale ses ouvrages où Catharine Macaulay est explicitement citée : Jacques-Pierre BRISSOT, Réplique de J. P. Brissot à Stanislas Clermont, concernant ses nouvelles observations sur les comités de recherches, sur les causes des troubles, les folliculaires, le long Parlement d'Angleterre, Paris, Desenne, 1790 ; JacquesPierre BRISSOT, Discours aux Jacobins, op. cit. ; Jacques-Pierre BRISSOT, « Discours du 8 août 1792 », dans les Archives parlementaires, Première série, Paris, Ed. Dupont, 1896, vol. 47, p. 575-576.

49. Dans sa correspondance, il fustige la corruption de la liberté anglaise à cause du luxe et de l'excessif pouvoir de la couronne. Honoré-Gabriel RIQUÉTI DE MIRABEAU, Mirabeau's Letters during his residence in England, London, Wilson, 1832, vol. 1, p. 316-317 ; vol. 2, p. 135-149. Pour ses rapports avec le mouvement radical anglais: Rachel HAMMERSLEY, The English republican tradition, op.cit. ,p. 174-175.

50. Honoré-Gabriel RIQUÉTI DE MIRABEAU, Mirabeau's Letters, op. cit., p. 145.

51. Lettre du 5 octobre 1786. Jacques-Pierre BRISSOT, Mémoires 1754-1793, op. cit., vol. III, p. 102.

52. Ibid. Italique dans le texte.

53. [Honoré-Gabriel RIQUÉTI DE MIRABEAU], Analyse des Papiers anglois, s.l., s.e., 1788, t. II, $n^{\circ}$ XXIX, 4-7 mars, p. 121. Lorsqu'il est en Angleterre, Mirabeau connait directement Macaulay.

54. Les premières critiques paraissent en octobre 1791: Journal des Savants, octobre 1791, p. 627-628 et Réimpression de l'ancien Moniteur, Paris, Plon, t. X, n² 282, 9 octobre 1791, p. 62.

55. L'idée de préparer une traduction française de The History of England remonte à 1777 et elle n'appartient pas à Mirabeau, mais au comte de Sarsfield. Rachel HAMMERSLEY, The English republican tradition, op. cit., p. 174. En revanche, il est plus difficile de partager l'idée avancée par François Quastana selon lequel Mirabeau lui-même aurait réalisé la traduction en 1777. François QUASTANA, La pensée politique de Mirabeau, op.cit., p.127. Dans une lettre sans date mais où Mirabeau parle de la publication de the History of Philip III de Robert Watson advenue en 1783, le futur constituant se réfère à la traduction comme d'un projet. Honoré-Gabriel RIQUÉTI DE MIRABEAU, Mirabeau's letters, vol. II., op. cit., p. 230. 
56. Il résume franchement son projet lorsqu'il écrit que « it is a affair of money. » Honoré-Gabriel RIQUÉTI DE MIRABEAU, Mirabeau's letters, vol. II., op. cit., p. 230 et 234-235.

57. Jacques-Pierre BRISSOT, Mémoires 1754-1793, op. cit., t. 1, p. 348.

58. Mirabeau avait déjà travaillé avec Durival pour la traduction de The History of the reign of Philip the Second, king of Spain de Robert Watson. Robert WATSON, Histoire du règne de Philippe II, roi d'Espagne. Ouvrage traduit de l'anglois par le comte de Mirabeau et J.-B. Durival, Amsterdam et Rotterdam, Changuion 1777-1778, 2 vol.

59. Mirabeau commence aussi à écrire des notes supplémentaires au texte de Macaulay, mais il s'arrête après les cent premières pages du premier volume. Elles sont sans doute son œuvre car, dans la préface du troisième volume, Guiraudet affirme s'être limité à traduire fidèlement l'ouvrage. Catherine macaulay, Histoire d'Angleterre, vol. III, op.cit., p.1-14 et Jacques C. VINCENS SAINT LAURENT, Charles-Philippe T. Guiraudet, dans Louis Gabriel Michaud (dir.), Biographie Universelle ancienne et moderne, Paris, Michaud, 1811-1862, t. XVIII [1817], p. 220-221. L'auteur de la notice biographique affirme que «c'est lui [Guiraudet] qui est le véritable auteur de la Traduction de l'Histoire de la Révolution d'Angleterre [sic] La preuve irrécusable de ce fait subsiste entre les mains de la famille de Guiraudet. » En revanche, il est plus problématique de dater précisément la réalisation effective de la traduction et de l'écriture du discours préliminaire ; mais tout laisse croire que les deux aient eu lieu en 1788. Dans un article consacré à Macaulay dans le journal Analyse des Papiers anglois, édité au début de l'année, Mirabeau cite tous les ouvrages dans les éditions originales sans faire référence à l'existence d'une traduction en cours. [Honoré-Gabriel RIQUÉTI De MIRABEAU], Analyses des Papiers anglais, cit, t. II, op.cit., p.123, 4-7 mars 1788. En plus, l'avis de l'éditeur Gattey et la brusque interruption des notes supplémentaires confirment l'hypothèse d'un travail mené près du déclanchement de la Révolution. Catherine MACAULAY, Histoire d'Angleterre, op. cit., vol. I, p. 8.

60. Le rôle éminent joué par Mirabeau à partir du printemps 1789 et l'absence de toute référence aux évènements révolutionnaires dans le discours introductif exclut une rédaction plus tardive.

61. L'œuvre est traduite par Jean-Baptiste Salaville à partir d'une version anglaise de la fin du $\mathrm{XVII}^{\mathrm{e}}$ siècle, sous le titre de [Anonyme], Théorie de la royauté après la doctrine de Milton, s.l. s.e., 1789. Comme l'on sait, la publication de ce texte connût plusieurs rebondissements car, à peine imprimé, il est retiré du commerce suite aux journées d'octobre. Il enfin imprimé au frais du département de la Drôme lors du procès du roi. Christophe TOURnu, Milton et Mirabeau, rencontre révolutionnaire, Paris, Edimaf, 2002, p. 19-24 et p. 45-46, Voir Pierre SERNA , « 1649-1799, le retour du refoulé ", op. cit.

62. Catherine MACAUlay, Histoire d'Angleterre, op. cit., vol. I, p. 9.

63. François QUASTANA, La pensée politique de Mirabeau, op. cit., p. 227 et sur Mirabeau historien de la dégénération du pouvoir royal : Ivi, p. 228-267.

64. Catherine macaulay, Histoire d'Angleterre, op.cit., vol. I, p. 106. Dans son introduction à l'histoire de la Franche Comté (jamais écrite), Mirabeau dénonce la soumission des historiens au joug monarchique. Honoré-Gabriel RIQUeTI DE MIRABEAU (Lucas DE MONTIGNY, dir.), Mémoires biographiques, littéraires et politiques de Mirabeau, écrits par lui-même, par son père, son oncle et son fils adoptif, Paris, vol. V, p. 327-330.

65. François FURET, Mona OZOUF, Deux légitimations historiques de la société française au XVIII ${ }^{e ̀ m e}$ siècle : Mably et Boulainvilliers, dans Annales. Économies, Sociétés, Civilisations. 34 année, $\mathrm{n}^{\circ} 3,1979$. p. 438-450 et, pour Boulainvilliers : Diego VENTURINo, Le ragioni della tradizione, Firenze, Le Lettere, 1993, notamment p. 3-26 et p. 252-305; sur Mably et plus en général sur les enjeux politiques de l'histoire dans la deuxième partie du siècle : Keith Michael BAKER, Au tribunal de l'opinion, op. cit., p. 45-84.

66. Hugh TREVOR-ROPE, History and Enlightenment, New Haven, Yale University, 2010, p. 2-12. La bibliographie sur le sujet est vaste, je signale ici des repères : Reinhart KOSELLECK, Le Règne de la 
Critique, Paris, Ed. Minuit, 1979 ; Jean-Marie Goulemot, Le règne de l'histoire, op. cit.; Giuseppe RICUPERATI (dir.), Historiographie et usages des Lumières, Berlin, Verl A. Spitz, 2002; Muriel BROT (dir.), Les philosophes et l'histoire au XVIII siècle, Paris, Herman, 2011 ; Chantal GRELL et Jean-Michel DUFAYS (dir.), Pratiques et concepts de l'histoire, Paris, Presses Universitaires de la Sorbonne, 1990.

67. Sur ce point, voir François FURET, Préface, dans Honoré-Gabriel RIQUETI DE MIRABEAU (François FURET, dir.), Discours, Paris, Gallimard, 1973, p. 11-13.

68. Catherine macaulay, Histoire d'Angleterre, op. cit., vol. I, p. 22. Dans une autre note il écrit : «Si l'ordre établi est mauvais, doit-on regarder comme constitutionnel l'usage qui l'empêche d'être bon ? Cet ordre fût-il même excellent, qu'elle autorité humaine peut empêcher une nation de le changer?»Ivi, p. 105.

69. Ivi, p. 112. En italique dans le texte. À propos du régicide, voir aussi Mirabeau, Théorie de la Royauté, op. cit., p. 50-56.

70. Sur ses positions politiques lors de la Révolution voir François FURET, Préface, dans HonoréGabriel RIQUETI DE MIRABEAU, Discours, op.cit., p. 15-23.

71. Ce silence s'explique, aussi, par une question d'opportunité politique. D'ailleurs, l'exemple du Commonwealth est source de débats même pour le mouvement radical tout au long du XVIII siècle. Rachel HAMMERSLEY, The English republican tradition, op.cit., p. 14-15; mais aussi et surtout Caroline ROBBINS, The Eighteenth-century Commonwealthman studies in the transmission, development and circumstance of English liberal thought from the Restoration of Charles II until the war with the Thirteen Colonies, Cambridge, Harvard University press, 1961; John Greville Agard POсоск, Le moment machiavelien, Paris, PUF, 1997; Blair WORDEN, Roundhead reputations, op.cit., p. 147-214 et Roger Charles RICHARDSON, The debate on the English revolution, op.cit., p. 40-62.

72. Sur le concept de républicanisme en Mirabeau: François QUASTANA, La pensée politique de Mirabeau, op.cit. Sur l'évolution du concept de républicanisme au XVIII ${ }^{\mathrm{e}}$ siècle: Keith Michael BAKER, «Transformations of Classical Republicanism in Eighteenth-Century France », dans Journal of Modern History, $\mathrm{n}^{\circ} 73, \mathrm{n}^{\circ} 1$, march 2001, p. 32-53.

73. Ces conclusions ne contredisent pas la lecture de la traduction offerte par Hammersley, qui la considère comme une forme de pression et d'avertissement adressé la royauté. Rachel HAMMERSLEY, The English republican tradition, op. cit., p. 180.

74. Catherine maCAUlay, Histoire d'Angleterre, op. cit., vol. I, p. 113.

75. Mirabeau exhorte les historiens à être «ces êtres privilégiés qui sentent que jamais rien ne pourra les détourner de l'intérêt de la justice et de la vérité [...] ; que ceux-là se livrent au noble instinct qui les anime [...] ; qu'ils instruisent les hommes, qu'ils détrompent les hommes, qu'ils écrivent pour des hommes." Honoré-Gabriel RIQUETI DE MIRABEAU (Lucas DE MONTIGNY, dir.), Mémoires biographiques, littéraires, op. cit., vol. V, p. 327-330.

76. Cela en dépit de l'admiration que Mirabeau avait pour le philosophe dans les années 1770. Ibid , p. 330.

77. Catherine MACAULAY, Histoire d'Angleterre, op. cit., vol. I, p. 123.

78. Ibid., p. 108.

79. Nonobstant Guiraudet est connu surtout pour sa traduction de l'œuvre de Machiavel, publiée pour la première fois en 1798 , il est un auteur actif au début de la Révolution et il participe à la vie politique de l'époque. En 1790, il est l'un des auteurs du Journal de la Société de 1789. Par la suite, il s'inscrit au club Feuillant. Sur sa vie, en dépit des imprécisions, Jacques C. VINCENS SAINT LAURENT, Pierre T. Guiraudet, dans Louis Gabriel MICHAUD (dir.), Biographie Universelle, op. cit., t. XVIII, p. 220-221.

80. Catherine MACAULAY, Histoire d'Angleterre, op. cit., vol. III, p. 18.

81. Catherine MACAULAY, Histoire d'Angleterre, op. cit., vol. III, p. 5.

82. Ibid, p. 24. 
83. Guiraudet dans un autre écrit attaque l'idée de transplanter l'exemple anglais en France. Charles Philippe GUIRAUDET, Explication de quelques mots importants de notre langue politique pour servir à la théorie de nos loix et d'abord de la loi constitutionnelle, Paris, s.e. 1792.

84. Catherine MACAULAY, Histoire d'Angleterre, op. cit., vol. III, p. 2.

85. Ivi, vol. I, p. 15-16.

86. Ivi, vol. III, p. 1 .

87. La faillite de Gattey est liée essentiellement à des motivations politiques, puisqu'il était l'éditeur de nombreuses publications contrerévolutionnaires, tels les Actes des Apôtres et la Gazette de Paris, qui sont suspendus après le 10 août.

88. Reinhart KOSELLECK, Le Futur passé, op. cit..

89. Pierre SERNA, « 1799, Le retour du refoulé... », op. cit., p. 219.

90. Jean-Marie GOULEMot, « Notes sur la mémorisation de la Révolution ... », op.cit., p. 17.

91. Pierre NORA, « Nation », dans François FURET et Mona OZOUF, Dictionnaire critique de la Révolution française, Paris, Gallimard, 1992, vol. II, p. 351.

92. Laurence Louis BONGIE, David Hume, Prophet of the Counter-revolution, op. cit., p. 64.

93. Blair WORDEN, Roundhead Reputations, op. cit., p. 1.

\section{RÉSUMÉS}

Quelques mois après la mort de Mirabeau est publiée à Paris la traduction de l'œuvre de Catherine Macaulay, History of England from the accession of James I to the Elevation of the House of Hanover, enrichie d'une longue préface de l'ancien tribun. Comment expliquer cet intérêt pour un récit historique qui avait suscité des vives polémiques en Angleterre ? Pourquoi le souvenir de la Guerre civile ressurgit-il au beau milieu d'une Révolution qui fonde toute sa légitimité sur la négation du passé ?

Ces questions structurent cet article dédié à la compréhension des usages politiques de l'histoire lors de la Révolution de 1789. Par la mise en miroir des nombreuses interventions qui se succèdent autour de l'œuvre de Macaulay, les pages qui suivent montrent l'importance du souvenir du XVII ${ }^{e}$ siècle anglais dans la construction d'un discours critique à l'égard de l'absolutisme royal et dans la formation intellectuelle d'acteurs politiques, tels que Brissot et Mirabeau. La traduction de 1791 révèle l'aboutissement d'un intérêt que les deux futurs révolutionnaires, comme toute leur génération, avaient développé à l'égard de l'histoire de la Guerre civile anglaise, pensée comme la première étape d'une histoire transnationale de la liberté.

A few months after the death of Mirabeau, the translation of History of England from the accession of James I to the Elevation of the House of Hanover of Catherine Macaulay is published in Paris with a long preface of the «tribun ». Why does this book about history of English Civil War reappear at the time of French Revolution? How explain such an interest for a historical work which provoked strong debates in England? This questions structure the present paper dedicated to the understanding of political uses of history in the Great Revolution of 1789. The analysis of papers on Macaulay's text shows the significance of English $17^{\text {th }}$ century memory in the construction of criticism of royal absolutism and of the thought of political players as Brissot and Mirabeau. The 1791 translation is the final achievement of a precocious interest which both revolutionary 
leaders have been for the history of English Civil war, considered as the first moment of transnational history of liberty.

INDEX

Mots-clés : guerre civile anglaise, républicanisme anglais, Catherine Macaulay, Jacques-Pierre Brissot, Honoré-Gabriel Mirabeau, Charles-Philippe Guiraudet

Keywords : English Civil War, English republicanism, Catherine Macaulay, Jacques-Pierre Brissot ; Honoré-Gabriel Mirabeau, Charles-Philippe Guiraudet 